\title{
BMJ Open Mii-vitaliSe: a pilot randomised controlled trial of a home gaming system (Nintendo Wii) to increase activity levels, vitality and well-being in people with multiple sclerosis
}

\author{
Sarah Thomas, ${ }^{1}$ Louise Fazakarley, ${ }^{1}$ Peter W Thomas, ${ }^{1}$ Sarah Collyer, ${ }^{2}$ \\ Sarah Brenton, ${ }^{2}$ Steve Perring, ${ }^{3}$ Rebecca Scott, ${ }^{4}$ Fern Thomas, ${ }^{1}$ \\ Charlotte Thomas, ${ }^{1}$ Kelly Jones, ${ }^{5}$ Jo Hickson, ${ }^{5}$ Charles Hillier ${ }^{2}$
}

To cite: Thomas $\mathrm{S}$,

Fazakarley L, Thomas PW, et al. Mii-vitaliSe: a pilot randomised controlled trial of a home gaming system (Nintendo Wii) to increase activity levels, vitality and well-being in people with multiple sclerosis. BMJ Open 2017;7:e016966. doi:10.1136/ bmjopen-2017-016966

- Prepublication history and additional material for this paper are available online. To view these files please visit the journal online (http://dx.doi. org/10.1136/bmjopen-2017016966).

Received 24 March 2017 Revised 5 May 2017 Accepted 8 May 2017

\section{CrossMark}

'Bournemouth University, Faculty of Health and Social Sciences, Bournemouth, Dorset, UK

${ }^{2}$ Poole Hospital NHS Foundation Trust, Dorset Multiple Sclerosis Service, Poole, Dorset, UK ${ }^{3}$ Poole Hospital NHS Foundation Trust, Medical Physics, Poole, Dorset, UK

${ }^{4}$ National Star College,

Cheltenham, Gloucestershire, UK

${ }^{5}$ Service user, Bournemouth, UK

Correspondence to

Dr Sarah Thomas;

saraht@bournemouth.ac.uk

\section{ABSTRACT}

Objectives While the health and well-being benefits of physical activity are recognised, people with multiple sclerosis (MS) often face greater barriers than the general population. The Nintendo Wii potentially offers a fun, convenient way of overcoming some of these. The aim was to test the feasibility of conducting a definitive trial of the effectiveness and cost-effectiveness of Mii-vitaliSe; a home-based, physiotherapist-supported Nintendo Wii intervention.

Design A single-centre wait-list randomised controlled study.

Setting MS service in secondary care.

Participants Ambulatory, relatively inactive people with clinically confirmed MS

Intervention Thirty participants were randomised to receive Mii-vitaliSe either immediately (for 12 months) or after a 6-month wait (for 6 months). Mii-vitaliSe consisted of two supervised Nintendo Wii familiarisation sessions in the hospital followed by home use (Wii Sports, Sports Resort and Fit Plus software) with physiotherapist support and personalised resources.

Outcomes Included self-reported physical activity levels, quality of life, mood, self-efficacy, fatigue and assessments of balance, gait, mobility and hand dexterity at baseline, 6 and 12 months. Interviews ( $n=25)$ explored participants' experiences and, at study end, the two Mii-vitaliSe facilitators' experiences of intervention delivery (main qualitative findings reported separately).

Results Mean (SD) age was 49.3 (8.7) years, 90\% female, with $47 \%$ diagnosed with MS $<6$ years ago and $60 \%$ new to active gaming. The recruitment rate was $31 \%(95 \% \mathrm{Cl}$ $20 \%$ to $44 \%)$. Outcome data were available for $29(97 \%)$ at 6 months and $28(93 \%)$ at 12 months. No serious adverse events were reported during the study. Qualitative data indicated that Mii-vitaliSe was well-received. Mean Wii use across both groups over the initial 6-month intervention period was twice a week for $27 \mathrm{~min} /$ day. Mean cost of delivering Mii-vitaliSe was £684 per person.

Discussion Mii-vitaliSe appears acceptable and a future trial feasible and warranted. These findings will inform its design.

\section{Strengths and limitations of this study}

- Used a randomised controlled trial design and included mixed methods and a consideration of long-term follow-up.

- Adherence to the Mii-vitaliSe intervention was assessed by daily completion of a log.

- Mii-vitaliSe incorporated home-based use, personalised support and behaviour change techniques.

- Physical outcome assessments were not undertaken blinded to group allocation.

- Feasibility focus paves way for a definitive trial of the effectiveness and cost-effectiveness of Mii-vitalise.

Trial registration ISRCTN49286846

\section{INTRODUCTION}

It is now widely recognised that physical activity provides many benefits for people with multiple sclerosis. ${ }^{12}$ These include improvements in physical fitness, mobility, muscle strength as well as possible secondary benefits such as improved quality of life, mood and well-being. ${ }^{3-7}$ Recent research suggests exercise is safe for people with MS with no increased risk of relapse or adverse events (AEs) ${ }^{8}$ However, people with MS typically are much less physically active than the general population. ${ }^{9}$ Keeping physically active can be challenging and people with MS face

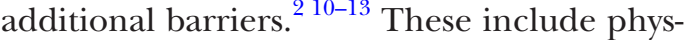
ical (eg, pain, fatigue, mobility and balance limitations, overheating), psychological (eg, fear, embarrassment or lack of confidence) and environmental (eg, transport, cost, lack of suitable facilities/trained staff) barriers. 
The Wii potentially overcomes some of these barriers by offering a relatively inexpensive, convenient, immersive and fun way to exercise in the home. ${ }^{14}{ }^{15}$ Recent physical activity guidelines for MS suggest working towards achieving $30 \mathrm{~min}$ of moderate aerobic activity twice a week and two sets of 10-15 repetitions of strength training exercises twice a week. ${ }^{16}$ However, as noted by de Souto Barreto, it is perhaps more important to get people who are currently inactive to do small amounts of activity even if they are not meeting guidelines for physical activity. ${ }^{17}$

While applications of the Wii are diverse and findings have been promising, ${ }^{14} 18$ the evidence base in MS is limited ${ }^{19}{ }^{20}$ with most studies small, preliminary and with limited follow-up. ${ }^{21-24}$ Nevertheless, early findings suggest the Wii is acceptable and enjoyable, ${ }^{25-27}$ safe for home use for those with mild MS symptoms, ${ }^{21}$ might be beneficial for balance ${ }^{24}{ }^{26}$ and fitness ${ }^{21}$ and potentially results in greater adherence than traditional rehabilitation programmes. ${ }^{28}{ }^{29}$ It has been suggested that active gaming might have a 'gateway effect' increasing individuals' confidence and encouraging and enabling them to engage in other physical activity. ${ }^{30}$

In a non-randomised controlled trial (using a baseline control period) involving a home-based Wii Fit programme of 14 weeks' duration, Plow and Finlayson reported that use of the Wii had declined at 14 weeks. ${ }^{21}$ They suggested that a more person-centred approach as well as the use of behaviour change techniques (such as motivational interviewing and problem solving) might help to improve longer term Wii use and foster intrinsic motivation. They highlighted the importance of considering individuals' functional levels, environment and preferences when prescribing a Wii-Fit programme. ${ }^{31}$

Findings from a scoping review of the exergaming literature suggest the need for mixed methods trials that incorporate long-term follow-up and follow patients from the clinical setting to the home. ${ }^{32}$ A recent systematic review of behaviour change interventions to increase physical activity in people with MS concluded further research is required focusing on dose, long-term impact and method of delivery. ${ }^{33}$ Qualitative work undertaken in the USA suggests that people with MS would like to be given more information about the benefits of exercise from their healthcare providers, along with materials to support home and community exercise and tools/strategies to support behaviour change. ${ }^{34}$

We have developed a physiotherapist-facilitated Wii intervention package that uses commercial software and aims to support people with MS to increase their physical activity levels ('Mii-vitaliSe') ${ }^{35}$ Our study design overcomes some of the limitations highlighted in the literature by incorporating home-based activity, behaviour change techniques with personalised support, employing mixed methods and including a consideration of long-term follow-up.

\section{Aim and objectives}

The main aim of this pilot study was to assess the acceptability and suitability of Mii-vitaliSe and to establish whether a definitive multicentre randomised controlled trial to assess effectiveness and cost-effectiveness is feasible and, if so, to inform its design and conduct.

The specific objectives of the study were:

1. Test procedures (including administration of the self-reported outcomes and the physical assessments, delivery of the intervention, recording and monitoring of AEs), estimate recruitment and retention rates, and refine the selection of outcome measures in preparation for a definitive randomised controlled trial.

2. Collect data on the variability of outcome measures to inform a sample size calculation for a larger trial.

3. Estimate adherence rates via a self-reported daily play $\log$ (in terms of frequency, intensity, duration).

4. Determine the acceptability of study processes such as randomisation and of the Mii-vitaliSe intervention to participants and obtain information about patterns of use/barriers to use.

5. Gather feedback from physiotherapists about their experiences of delivering Mii-vitaliSe.

6. Pilot a measure of healthcare resource use for a future economic evaluation.

\section{METHODS}

\section{Ethics and safety monitoring}

The trial was overseen by a steering committee and is reported in accordance with the Consolidated Standards of Reporting Trials guidelines for pilot and feasibility studies. ${ }^{36}$ The study was reviewed and given a favourable opinion by the National Health Service (NHS) South Central Hampshire B Research Ethics Committee (ref: 12/SC/0420). All participants gave written informed consent before taking part. Poole Hospital NHS Foundation Trust was the study sponsor. A Safety Monitoring Committee comprising two independent advisors (a neurologist with expertise in MS and clinical trials and a physiotherapist with expertise in risk assessment and management of service users with neurological disability) reviewed any reported AEs and made recommendations to the study steering group.

\section{Study design}

This mixed methods pilot study used a single-centre wait-list randomised controlled design. ${ }^{35}$ Participants were randomised to receive Mii-vitaliSe (plus usual care) immediately (immediate group) or after a 6 -month delay (delayed group). Those in the immediate group received Mii-vitaliSe for approximately 12 months and those in the delayed group for approximately 6 months following a 6-month delay (during the delay they were asked to refrain from using the Wii). This design permitted a preliminary consideration of long-term (12 months) follow-up (immediate group only) (see Figure 1). 


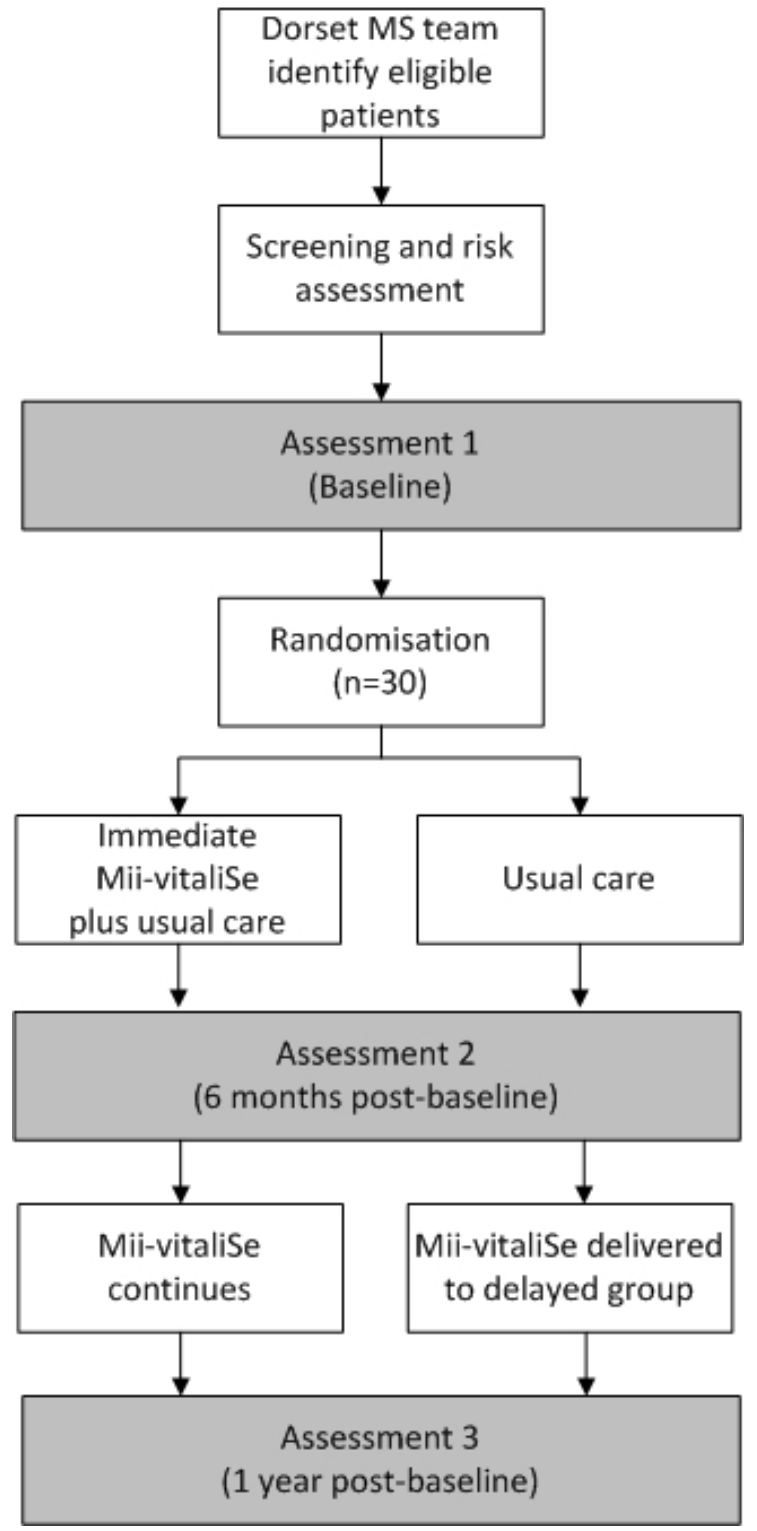

Figure 1 Study design.

Semi-structured interviews (face-to-face at home) were undertaken by the study researcher with a purposive sample of participants at 6 and 12 months follow-up and at the end of the study with the two physiotherapists who delivered Mii-vitaliSe (study objectives 4 and 5).

A feasibility economic component was included to pilot the use of a healthcare resource use questionnaire and to capture costs related to delivering Mii-vitaliSe (study objective 6 ).

\section{Participants}

The main inclusion criteria were (i) a clinically definite diagnosis of MS; (ii) aged 18 years or above; (iii) satisfied a risk assessment (see below); (iv) relatively physically inactive (active for a period of $30 \mathrm{~min}$ or more on fewer than 5 days per week; (vi) having a suitable television at home.

The main exclusion criteria were (i) Adapted Patient Determined Disease Steps (APDDS) Scale ${ }^{37}$ score of 1 or $\geq 6$ (equivalent to an Expanded Disability Status Scale score of 1 or $\geq 6$; (ii) a relapse within the past 3 months that required treatment with corticosteroids and/or a hospital admission; (iii) already participating in exercise or rehabilitation research; (iv) a medical condition placing an individual at risk from exercise participation; (vi) owns a Wii and is currently using it on a weekly basis or more; (vii) unwilling or unable to comply with the protocol (eg, long vacation planned). The full eligibility criteria are described in the published protocol. ${ }^{35}$

\section{Sample size}

As this was a pilot study designed to examine methodological and procedural uncertainties of a future full-scale trial, we did not undertake a formal sample size calculation for clinical effectiveness. We used a sample size of 30 , which is a common rule of thumb for pilot studies ${ }^{38}$ and adequate for the study objectives involving estimation of the recruitment rate, estimating the SD of potential primary outcomes and estimating adherence to the intervention (for further details see published $\operatorname{protocol}^{35}$ ).

\section{Recruitment}

Recruitment took place between February 2013 and July 2013 with the last follow-up conducted in August 2014. Participants were recruited via multidisciplinary team members of an MS Service in a secondary care setting (Poole Hospital NHS Foundation Trust). Members of the team screened potential participants using a checklist and, if they were eligible, either gave or sent them an invitation letter with a simple one page summary ('Key Facts') about the study, participant information sheets, the APDDS Scale, ${ }^{37}$ a brief question about current levels of physical activity, a reply slip and pre-paid envelope. Those interested in participating or finding out more were asked to return the reply slip along with their responses to the questions. A follow-up reminder was sent with another copy of the information pack if no response was received.

\section{Screening and risk assessment}

The study researcher (a senior lecturer in physiotherapy) telephoned those expressing interest in the study, described the study in more detail, answered any questions and went through the remaining screening criteria. Those eligible were then visited at home for a risk and home suitability assessment undertaken by the study researcher. This involved an assessment of (i) ability to maintain standing balance with eyes open for 1 min on the balance board; (ii) balance reactions and ability to step off safely from the balance board forwards, sideways and backwards; (iii) assessment of the home environment with minor environmental modifications suggested (if appropriate/possible). If participants satisfied the risk assessment criteria, they were asked to provide informed written consent and were then enrolled into the study. 


\section{Blinding}

The nature of the intervention meant that participant blinding was not possible. The study researcher (not blinded to allocation) completed the physical assessments along with a clinical scientist (blinded to allocation). All other outcomes (with the exception of the activPAL accelerometer) were self-reported and administered postally (see 'Outcome measures' section for further detail).

\section{Study interventions}

Mii-vitaliSe

The format and content of Mii-vitaliSe is summarised in the published protocol and its development is described in brief elsewhere. ${ }^{39}$ A consultation workshop was held to refine and optimise the Mii-vitaliSe intervention package. The workshop was attended by eight people with MS with experience of using the Wii and four health professionals from the research team (three physiotherapists and one psychologist). Table 1 summarises the timing, content and mode of delivery of Mii-vitaliSe. The rationale of Mii-vitaliSe is to support people with MS to increase activity levels in their own homes using the Nintendo Wii. Mii-vitaliSe encourages the internalisation of goals, and aims to provide individuals with skills, strategies and support to identify solutions to overcome barriers they encounter. The intervention draws on relevant psychological frameworks and theories (motivational interviewing, ${ }^{40}$ social cognitive, ${ }^{41-43}$ cognitive behavioural ${ }^{44}$ and self-determination theory ${ }^{45}$ ) and incorporates behaviour change techniques.

A Mii-vitaliSe handbook (29 pages) provided information about the benefits of physical activity and tips and advice for using the Wii safely and maintaining a physical activity programme, including quotations from people with MS with experience of using the Wii.

The intervention was personalised and this was achieved by the provision of regular one-to-one support from a physiotherapist (face-to-face and telephone) and a personal activity workbook (36 pages) that facilitated individualised goal setting, feedback, action and coping planning and monitoring of progress (Table 1). Participants were also provided with a 'Play Log' to encourage self-monitoring of activity/progress (see 'Process measures' section) and a set of descriptors of all the software games with space for notes/comments.

Following the risk assessment in the home and two orientation sessions in the hospital, the Wii console was set up in participants' homes. Participants were provided with commercially available software (Wii Fit Plus, Wii Sports and Wii Sports Resort) along with the Wii balance board (and non-slip cover), two Wii remote controls, two Nunchuk controls, battery and remote control chargers and spare rechargeable batteries. All participants were given a copy of the Mii-vitaliSe handbook and personal activity workbook presented in a ringbound folder.

The intervention was delivered by two Mii-vitaliSe facilitators (both senior physiotherapists). The study researcher undertook training in motivational interviewing (MI) and oriented the two Mii-vitaliSe facilitators to the MI approach.

\section{Usual care}

The Dorset MS Service provides multidisciplinary support. Patients are reviewed annually by the team at an outpatient clinic or home visit appointment. On completion of the review and necessary assessments, medical and therapy treatments are delivered as required. If patients experience a deterioration of their symptoms before the next review they can self-refer to the service. Education, support and advice regarding disease modifying therapies, management of symptoms and carer support is available from the specialist nurse. The team operates a helpline service Monday to Friday and messages can be left on an answer-phone outside the scheduled helpline hours.

\section{Randomisation}

To ensure good allocation concealment, random allocation was email-based and administered by the study statistician. Randomisation was carried out in a one-to-one ratio and the sequence was produced using a computer-based random sequence generator. Variable-sized blocks were used to ensure approximately equal numbers in the two arms. No stratification was used.

\section{Descriptors and outcome measures}

At baseline, we gathered the following demographic and clinical descriptors: age, sex, ethnicity, disease type, time since diagnosis, relapses requiring corticosteroids and/or hospital admission, self-reported mobility (APDDS score), education, employment status, marital status, household composition.

As this was feasibility work, a broad range of outcomes was included encompassing physical activity, physical functioning, quality of life, mood and self-efficacy. Outcome measures were assessed at baseline and at 6 and 12 months. Physical assessments were undertaken in the hospital setting and administered by the study researcher and a clinical scientist at similar times of the day wherever possible to reduce the confounding effect of fatigue. Self-reported questionnaires (presented in a large font in a booklet format) were completed by participants in their homes at their own convenience and pace. For a more detailed description of outcome measures, see online web supplement 1 .

\section{Adverse events}

All AEs and their possible relation to the Mii-vitaliSe intervention were closely monitored, documented and reported to the Study Safety Monitoring Committee. AE data were gathered via multiple sources: from the Dorset MS team, at physical assessment hospital visits, from the Mii-vitaliSe physiotherapists via face-to-face/telephone contacts with participants, via daily play logs (see 'Outcome measures' section) and during the qualitative interviews. These were collated by the study researcher and reviewed by the independent Safety Monitoring 


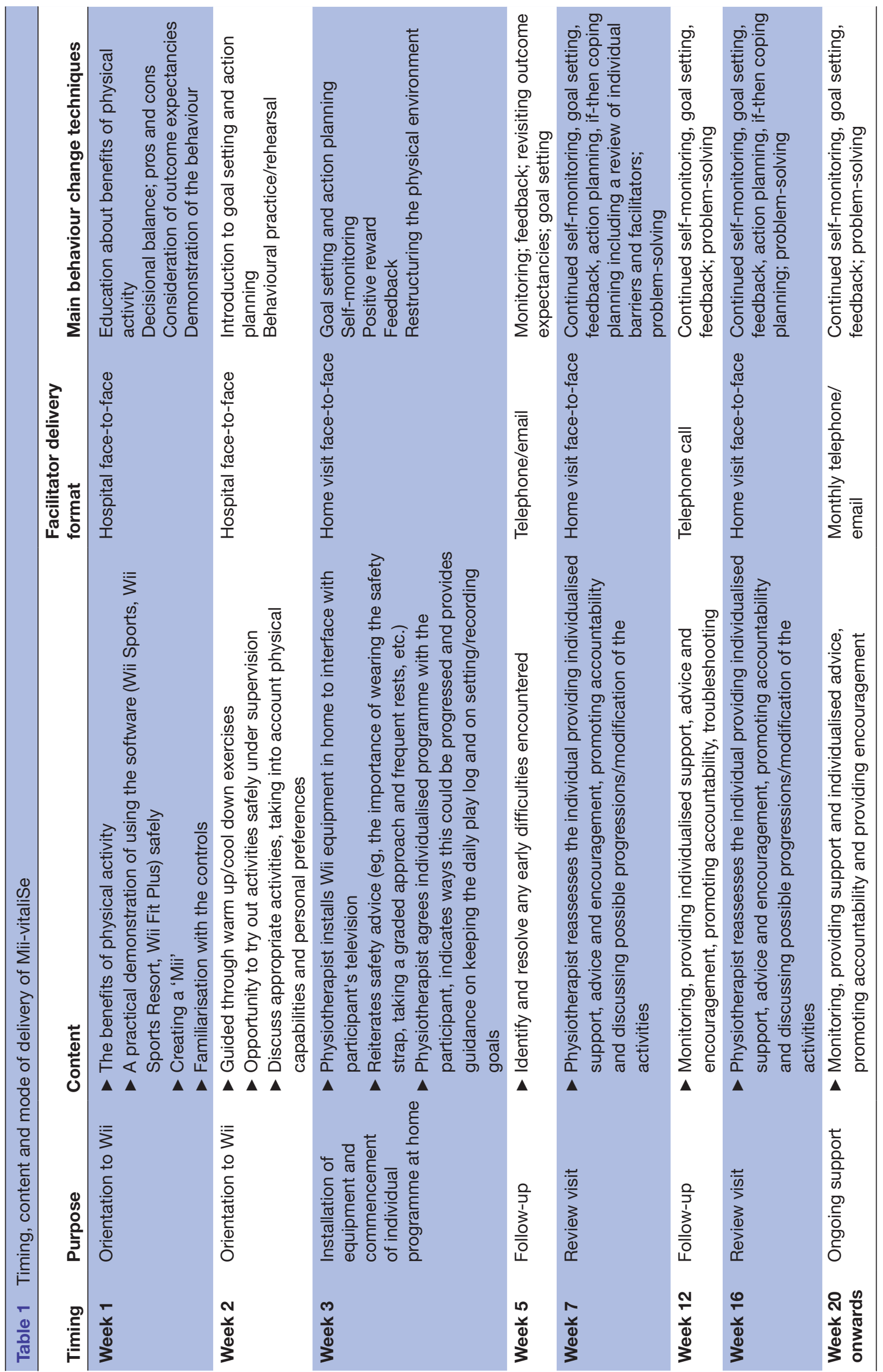


Committee. The Chief Investigator assessed each AE to establish if it should be classified as a serious adverse event (SAE) according to the National Research Ethics Service definition (in terms of seriousness, relatedness and unexpectedness). As we were interested in the feasibility and safety of the intervention, we gathered detailed information about AEs that were linked to Wii use even if these were expected and relatively minor (such as muscle ache, discomfort, pain).

\section{Physical activity}

\section{Godin Leisure-Time Exercise Questionnaire (GLTEQ) 4647}

This is a self-reported measure of usual physical activity that has been widely used in MS research. It consists of two items: the first measures frequency and intensity of exercise during free time in a typical week. These weekly frequencies are multiplied by metabolic equivalents and summed to form a measure of total leisure activity. The second question asks about the frequency of engaging in any regular activity long enough to work up a sweat with three response options provided (often, sometimes, never/rarely).

\section{activPAL3 tri-axial accelerometer ${ }^{48} 49$}

This classifies an individual's free-living activity into periods spent sitting, standing and walking and gives the number of steps and sit-to-stand episodes. At each assessment point participants were instructed to wear the activPAL for 24 hours/day for 7 consecutive days but to remove the device during water-based activities (showering, bathing, swimming, etc). We did not specify which leg to attach it to as people could have had weakness in one leg.

\section{Psychological well-being, quality of life}

\section{Hospital Anxiety and Depression Scale (HADS) ${ }^{50}$}

The HADS is a self-report measure that incorporates an anxiety and a depression subscale. Each subscale consists of seven items with a 4-point Likert-type response scale. Higher scores indicate greater levels of anxiety and/or depression.

\section{EuroQol-5 Dimensions-5 Levels (EQ-5D-5L) $)^{51}$}

This is a measure of health status consisting of a descriptive system (five dimensions: mobility, self-care, usual activities, pain/discomfort, anxiety/depression). Each dimension has five levels (no problems, slight problems, moderate problems, severe problems and extreme problems). We converted participants' responses to utility values using a value set for England. ${ }^{52}$ Additionally, a visual analogue scale records respondents' selfrated health on a scale with end points labelled 'the best health you could imagine' and 'the worst health you can imagine'.

\section{Multiple Sclerosis Impact Scale (MSIS-29) $)^{53}$}

This scale measures the physical (20 items) and psychological impact (nine items) of MS on day-to-day life. It uses 5-point Likert-type response scales ranging from 'not at all' to 'extremely' and is based on quality of life in the last 2 weeks.

\section{The Fatigue Symptom Inventory (FSI) $)^{54}$}

The Fatigue Symptom Inventory is a 14-item self-report multidimensional questionnaire which measures the severity (four items), frequency (two items) and diurnal variation of fatigue (one item) and its perceived interference on quality of life (seven items). In the current study we report the severity and interference items and an interference summary score.

\section{The Medical Outcomes Short-Form Survey V.2 (SF-36 v.2) ${ }^{55}$}

The SF-36 v.2 measures eight dimensions of quality of life: physical functioning, role limitations because of physical health problems, bodily pain, general health perceptions, vitality, social functioning, role limitations because of emotional problems and mental health. In the current study we report only the Physical Component Summary (PCS) and the Mental Component Summary (MCS) scores. We also derived SF-6D utility scores from the SF- $36 .^{56}$

\section{Self-efficacy}

The Spinal Cord Injury Exercise Self-Efficacy Scale (SCI-ESES ${ }^{57}$

This scale was originally developed and validated in spinal cord populations. However, it has since been used in MS. ${ }^{11}$ It consists of 10 items that ask respondents to indicate their confidence in performing physical activities and exercise, with higher scores indicating higher perceived self-efficacy.

\section{The Multiple Sclerosis Self-Efficacy (MSSE) Scale $e^{58}$}

This 18-item scale comprises two subscales: (i) confidence with function and (ii) confidence with ability to manage symptoms/cope with the demands of illness. In the current study, we only used the latter 9-item subscale.

\section{Balance/gait/mobility}

Two-Minute Walk Test (2MWT) $)^{59}$

This is a measurement of walking endurance by assessing walking distance over $2 \mathrm{~min}$.

\section{Step Test ${ }^{00}$}

This is a test of dynamic standing balance. It involves recording the number of times a participant steps one foot fully on, then off, a $7.5 \mathrm{~cm}$ and $15 \mathrm{~cm}$ block as quickly as possible in two separate $15 \mathrm{~s}$ time periods, one for each foot leading.

\section{Steady Stance Test ${ }^{11}$}

The steady stance test measures the ability of an individual to maintain a steady stance for a $60 \mathrm{~s}$ period in five predetermined stances without support (feet apart, feet together, stride stance, tandem stance, single leg stance).

\section{Instrumented Timed Up and Go (i-TUG) ${ }^{62}$}

This is a timed test used to examine functional mobility and requires the individual to stand up from a seated position, walk $3 \mathrm{~m}$, turn, walk back and sit down. The 
accelerometer and telemetry system were attached to the participant's back with a strap. Three-axis accelerometer data were recorded in real-time on a PC via telemetry. Participants undertook one practice run followed by two recorded performances of the instrumented timed up and go test. Time to undertake the test was derived from the accelerometer data. Mean performance across the two recorded timed tests was used in the analysis.

\section{Gait Stride-time Rhythmicity ${ }^{63}$}

This was measured using a portable recorder connected to flat in-shoe heel impact sensors. Participants were asked to walk unaccompanied on an extended walk in a non-laboratory setting along a covered and flat route (between 200 and 250 steps per foot). They were instructed to walk at their normal pace without interruption and could use their normal orthotic or walking aid. The stride time between adjacent heel strikes was recorded for each foot separately and the mean and SD stride times were calculated.

\section{Static Posturography $y^{64}$}

The Poole Hospital Static Posturography System provided a method for objectively assessing balance via the tracking of limits of sway in a series of standardised conditions. It used ultrasound (transmitter was attached to the participant's waist) time-of-flight posturography measurements to locate a participant's centre of gravity $(\mathrm{CoG})$ in a protocol mirroring the Equitest Sensory Organisation Test protocol. ${ }^{65}$ The motion of the CoG was accurately tracked during the course of $20 \mathrm{~s}$ assessment periods as the participant stood on either a solid or soft surface with eyes open or eyes closed or when doing a cognitive distractor task. We report the Equilibrium Quotient per cent score (100-(anterior posterior maximum sway during $20 \mathrm{~s} /$ maximum anterior posterior movement possible without losing balance) $\times 100$ ). We note that the Wii balance board has been used as a tool for posturography assessment. ${ }^{146667}$

\section{Hand dexterity/coordination}

Nine-Hole Peg Test (9HPT) ${ }^{68}$

This timed test assesses finger and hand dexterity in both hands. It involves placing nine pegs into a peg board and then removing them as quickly as possible.

\section{Process measures}

\section{Daily play log}

This had a dual purpose of providing a means for participants to self-monitor their Wii use as well as providing the research team with insights into levels and patterns of use. It included the following fields: date, whether played, adverse events (eg, pain, tenderness, soreness, fatigue, dizziness, headaches, aching, stiffness, falls or near falls), games played, total duration and number of bouts of activity, intensity, ${ }^{69} 70$ enjoyment and fatigue rating (on a scale of 1-10), reasons for non-use, free text comments. As we had concerns that asking people to complete a daily log might be onerous, we also tried out an alternative, briefer $\log$ for those in the immediate group from months 6 to 12 . This was a calendar format which simply required participants to either put a cross on the date to indicate non-use or to write down the duration played if they had used the Wii.

We originally envisaged that adherence would be operationalised as the total number of days on which activities were performed divided by the number of days agreed with the physiotherapist in a participant's action plan. However, it became apparent that given our long-term timescales, the variability of MS and the frequent need to adjust goals (eg, to progress them or reduce them due to symptom fluctuations) that this definition was not practical. Instead we measured daily use (in terms of frequency, duration and intensity) or non-use use of the Wii. Play logs were collected from participants in the immediate group at the end of 6 months, and at 12 months from the delayed group. Due to resource and time constraints, we were not able to collect any data directly from the Wii consoles.

\section{Resource use and intervention costs}

We piloted a self-reported healthcare resource use questionnaire that we have used previously in a trial of MS fatigue. ${ }^{71}$ This questionnaire was administered at 6 and 12 months postbaseline. As we were primarily interested in the feasibility of collecting these data and their completeness, we present these data descriptively only. Intervention costs for delivery of Mii-vitaliSe are based on within-study data including work sampling forms completed by the Mii-vitaliSe facilitators to record preparation, contact and travel time. Facilitator costs were estimated using pounds sterling at $2013{\text { unit } \text { costs. }^{72}}$

\section{Interviews}

Interviews $(\mathrm{n}=25)$ were conducted by the study researcher in people's homes- 13 were undertaken at 6 months ( 7 immediate and 6 delayed group) and 12 at 12 months (6 immediate and 6 delayed group). These explored participants' experiences of study participation and of receiving Mii-vitaliSe. The study researcher also conducted interviews at the study end with the two physiotherapists who had delivered Mii-vitaliSe. In this article, we focus on the acceptability of study processes, study design and of the Mii-vitaliSe intervention. The main findings from the qualitative component relating to barriers and facilitators to using the Wii will be reported in depth elsewhere.

\section{Statistical analysis}

The analysis was undertaken using SPSS V.21. As this was a pilot study, the analysis is mainly descriptive. ${ }^{38}$ Estimates of recruitment and retention rates and SDs of baseline potential primary outcomes are presented with 95\% CIs. Preliminary effect size estimates with 95\% CIs are presented in order to inform our consideration of potential primary outcome measures for the future trial and the plausibility of effect sizes. Standardised effect sizes (Cohen's $d$ ) were obtained by dividing effect sizes 
by pooled baseline SD. Participants were analysed in the group they were randomised to and we attempted to collect outcome measures on everyone randomised. Missing data were assumed to be missing completely at random and no imputation methods were used. Analysis of covariance was used to estimate effect size for each outcome variable at 6 and 12 months follow-up, adjusting for their baseline values.

Adherence rates were estimated via the data from the play log. This was done in two different ways: (a) using day as the unit of analysis (ie, percentage of days used) and (b) using participant as the unit of analysis (ie, percentage of days of use for each participant was calculated and then averaged over all participants). Where a play log or calendar was not completed for one or more days, we made the conservative assumption that the Wii was not used; thus, Wii use might be underestimated. Wii use towards the end of the 12-month period was calculated from the last 30 days of follow-up in the immediate group. Descriptive statistics were used to summarise participants' use of the Wii, including which games were used, intensity of exercise, time of day, reasons for not using the Wii and if they played the Wii, whether this was alone or with others.

Visual inspection of the 'Activity Summary' output by day and week provided by the activPAL software programme (V.7.2.32) was used to provide an estimation of the number of valid wear days for each participant per 7-day administration period.

\section{Qualitative analysis}

The semi-structured interviews were transcribed verbatim and analysed thematically using a framework method. ${ }^{73}$ Two researchers (ST, LF) familiarised themselves with the transcripts and developed an agreed coding scheme and analytical framework combining a priori issues from the original topic guide and emerging themes. These codes were then charted in a matrix. Data relating to the acceptability of the outcome measures were content analysed and summarised descriptively.

\section{RESULTS}

\section{Study procedures, recruitment and retention rates}

The planned recruitment target of 30 people randomised was achieved. The recruitment rate was $31 \%$ (95\% CI $22 \%$ to $41 \%$ ) with thirty people randomised of the 98 who had been sent information packs (see Figure 2). We cannot comment on reasons for non-participation as the NHS Research Ethics Committee recommended that we did not use a decline slip in the study.

There were two dropouts in the immediate Mii-vitaliSe arm (for medical reasons) and these participants had incomplete follow-up data. Thus, 29 (97\%) (95\% CI $83 \%$ to $99 \%$ ) participants had complete self-reported questionnaire follow-up data at 6 months and 28 (93\%) (95\% CI $79 \%$ to $98 \%$ ) at 12 months.

\section{Baseline characteristics}

Ninety per cent of participants were female and $70 \%$ had a diagnosis of relapsing remitting MS. Mean (SD) age was 49 (8.7) years with $47 \%$ diagnosed with MS for $<6$ years. Sixty per cent of the sample had not used a home gaming system before (see Table 2 and 3 ).

\section{Outcome measures}

As this was a pilot study, our focus is on presenting descriptive data. ${ }^{38}$ We do, however, present preliminary estimates of effect size to inform our consideration of potential primary outcome measures.

Questionnaire booklet return rates were high. Each participant was administered a self-report questionnaire booklet on three separate occasions. Two participants withdrew from the study for medical reasons and were not sent questionnaires after they withdrew. Eightyseven questionnaires were returned completed (97\% of the planned assessment occasions and $100 \%$ of those administered).

Overall, rates of missing data for the self-reported outcomes were low. Anecdotal comments made by several participants during the telephone screening and feedback from the semi-structured interviews suggested that some participants found it difficult to complete the APDDS Scale as they felt they did not fit into a single category. When completing the Godin Leisure-Time Exercise Questionnaire, participants sometimes left blank spaces rather than putting zeros $(\sim 10 \%$ of responses $)$. In this instance, if participants had completed at least one of the three weekly frequency items but left one or both of the others blank (and had completed other questionnaires), we assumed they intended zero. However, in a future trial it will be important to emphasise more strongly that a response should be made to all items, even if that response is zero.

The interview data indicated that while participants found the physical assessments undertaken in the hospital interesting and acceptable, they were quite tiring and it was sometimes inconvenient to have to travel to the hospital, particularly for those working full-time.

Table 4 presents descriptive statistics for candidate outcomes for a future definitive trial. Descriptive statistics for all other outcomes included in this feasibility study are presented in online supplementary Tables S1-S5. For the self-reported outcome measures all standardised effect sizes (SES) at 6 months were in the direction of benefit for the immediate group with the exception of those for the SF-36 PCS, the EQ-5D-5L VAS and derived single index value, the NHPT and $4 / 5$ of the reported outcomes of the FSI. All the balance and gait measures produced SES in the direction of benefit. However, the CIs were wide and spanned zero.

\section{activPAL data}

Overall of 90 possible measurement occasions there were no data available for $6(7 \%)$ occasions (baseline, $\mathrm{n}=2 ; 6$ months, $\mathrm{n}=1 ; 12$ months, $\mathrm{n}=3$ ). Of the 84 measurement 


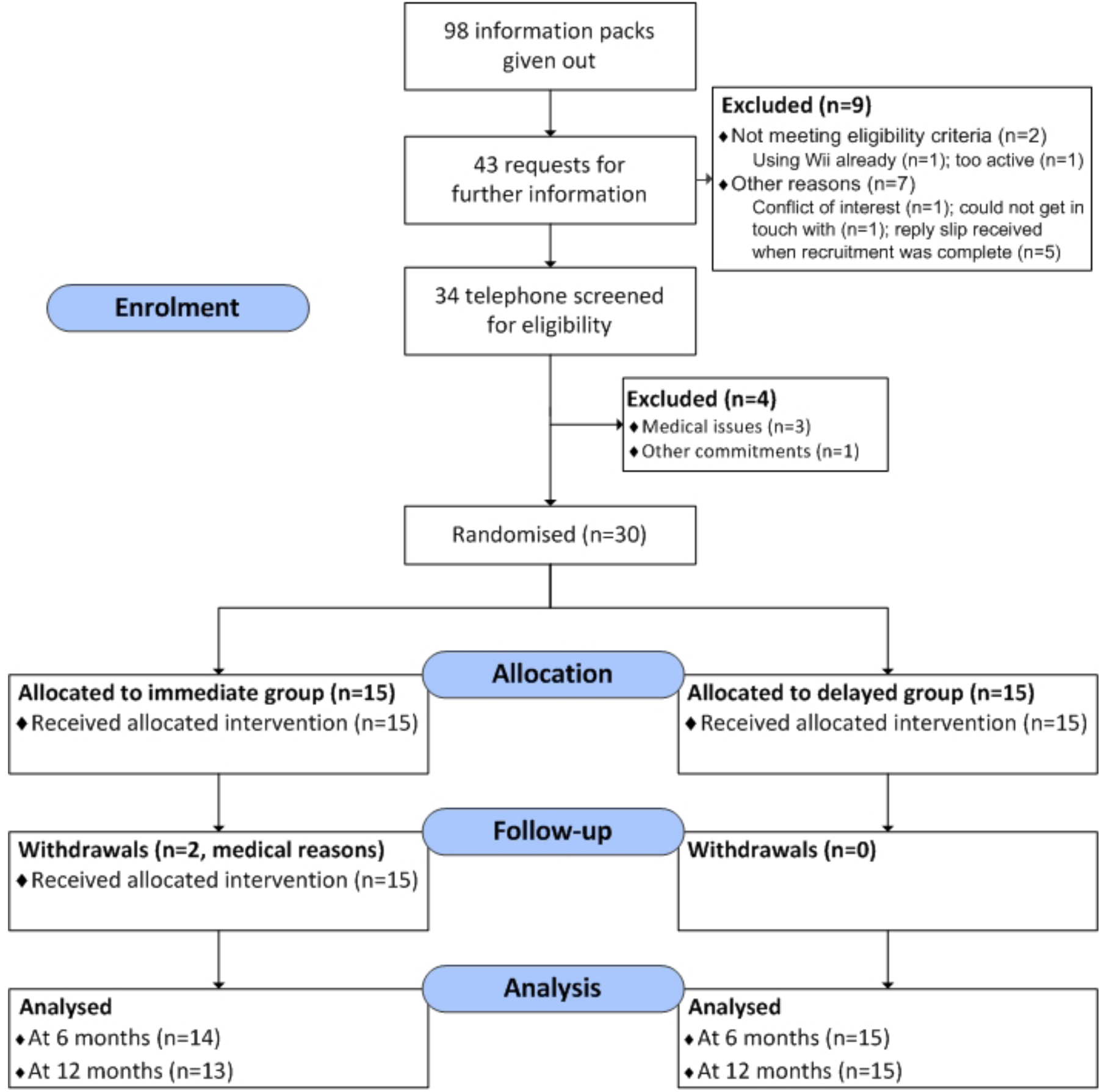

Figure 2 Consolidated Standards of Reporting Trials (CONSORT) diagram.

occasions with data, 65 had a full 7 days, 18 had 6 days and 1 had 5 days, Out of a maximum 630 days' data (7 days for each of 90 measurement occasions), data were available for $90 \%$. Fourteen participants $(47 \%)$ had the full 7 days' data available for each measurement occasion and $24(80 \%)$ had at least 6 days' data (see online web supplement 2, Table S6).

Some participants reported that if they attached the activPAL device using PAL stickies only (with no medical tape), the activPAL fell off their leg in bed at night and when removing clothes. We have contacted activPAL for advice and they recommended using a Nitrile sleeve in combination with waterproof tape to ensure a continuous overnight recording and to make the device water resistant (so it can be worn in the shower). In the current study, we did not specify the leg on which to attach the activPAL. In a future trial, we would ask people to wear the activPAL on their stronger leg if there are differences in functioning.

\section{Adverse events}

During the study period, there were no SAEs reported. The following Wii Fit Plus-related AEs were logged during the trial or reported in the 12-month interviews: 
Table 2 Descriptive statistics for demographic and baseline characteristics of participants

\begin{tabular}{|c|c|c|c|}
\hline & $\begin{array}{l}\text { Immediate } \\
(n=15)\end{array}$ & $\begin{array}{l}\text { Delayed } \\
(n=15)\end{array}$ & $\begin{array}{l}\text { Total } \\
(n=30)\end{array}$ \\
\hline \multicolumn{4}{|l|}{ Gender (n (\%)) } \\
\hline Female & 14 (93\%) & $13(87 \%)$ & $27(90 \%)$ \\
\hline Male & $1(7 \%)$ & $2(13 \%)$ & $3(10 \%)$ \\
\hline \multicolumn{4}{|l|}{ Age (years) } \\
\hline Mean (SD) & 50.9 (8.08) & $47.6(9.26)$ & $49.3(8.70)$ \\
\hline Range & $37-63$ & $33-65$ & $33-65$ \\
\hline \multicolumn{4}{|l|}{ Ethnicity } \\
\hline White English & $14(93 \%)$ & $13(87 \%)$ & $27(90 \%)$ \\
\hline White other British & $1(7 \%)$ & 0 & $1(3 \%)$ \\
\hline Other white & $0(0 \%)$ & $2(13 \%)$ & $2(7 \%)$ \\
\hline \multicolumn{4}{|l|}{ Disease type (n (\%)) } \\
\hline Benign & $0(0 \%)$ & $1(7 \%)$ & $1(3 \%)$ \\
\hline Relapsing-remitting & $12(80 \%)$ & $9(60 \%)$ & $21(70 \%)$ \\
\hline Secondary progressive & $3(20 \%)$ & $2(13 \%)$ & $5(17 \%)$ \\
\hline Primary progressive & $0(0 \%)$ & $1(7 \%)$ & $1(3 \%)$ \\
\hline Participant states 'do not know' & $0(0 \%)$ & $2(13 \%)$ & $2(7 \%)$ \\
\hline \multicolumn{4}{|l|}{ Years since diagnosis } \\
\hline$<1$ year & $1(7 \%)$ & $2(13 \%)$ & $3(10 \%)$ \\
\hline $1-5$ years & $7(47 \%)$ & $4(27 \%)$ & $11(37 \%)$ \\
\hline $6-10$ years & $3(20 \%)$ & $4(27 \%)$ & $7(23 \%)$ \\
\hline $11-15$ years & $2(13 \%)$ & $1(7 \%)$ & $3(10 \%)$ \\
\hline$>16$ years & $2(13 \%)$ & $4(27 \%)$ & $6(20 \%)$ \\
\hline $\begin{array}{l}\text { Relapse in previous } 6 \text { months* }(\mathbf{n}(\%)) \\
\text { *requiring corticosteroids or hospital admission }\end{array}$ & $0(0 \%)$ & $0(0 \%)$ & $0(0 \%)$ \\
\hline \multicolumn{4}{|l|}{$\begin{array}{l}\text { Adapted Patient Determined Disease Steps (APDDS) } \\
\text { score }\end{array}$} \\
\hline 2 (noticeable symptoms, but mild) & $3(20 \%)$ & $2(13 \%)$ & $5(17 \%)$ \\
\hline 3 (limitations with Activities of Daily Living (ADLs)) & $0(0 \%)$ & $1(7 \%)$ & $1(3 \%)$ \\
\hline 4 (interferes with walking, can walk 300-500 m) & $5(33 \%)$ & $2(13 \%)$ & $7(23 \%)$ \\
\hline 5 (can walk 100-200 m but often use a stick) & $6(40 \%)$ & $10(67 \%)$ & $16(53 \%)$ \\
\hline 6 (need a stick or single crutch) $\dagger$ & $1(7 \%)$ & $0(0 \%)$ & $1(3 \%)$ \\
\hline \multicolumn{4}{|l|}{ Educationt (n (\%)) } \\
\hline \multicolumn{4}{|l|}{ †Highest qualification achieved: } \\
\hline No qualifications & $2(13 \%)$ & $0(0 \%)$ & $2(7 \%)$ \\
\hline One or more GCSE (or equivalent) & $2(13 \%)$ & $4(27 \%)$ & $6(20 \%)$ \\
\hline One or more $\mathrm{A}$ level (or equivalent) & $1(7 \%)$ & $2(13 \%)$ & $3(10 \%)$ \\
\hline First degree (or equivalent) & $2(13 \%)$ & $3(20 \%)$ & $5(17 \%)$ \\
\hline Higher degree/professional qualification & $2(13 \%)$ & $2(13 \%)$ & $4(13 \%)$ \\
\hline Other & $6(40 \%)$ & $4(27 \%)$ & $10(33 \%)$ \\
\hline \multicolumn{4}{|l|}{ Employment status } \\
\hline In paid employment (>30 hours per week) & $6(40 \%)$ & $3(20 \%)$ & $9(30 \%)$ \\
\hline In paid employment ( $\leq 30$ hours per week) & $4(27 \%)$ & $5(33 \%)$ & $9(30 \%)$ \\
\hline Self-employed & $0(0 \%)$ & $1(7 \%)$ & $1(3 \%)$ \\
\hline $\begin{array}{l}\text { Not in paid employment (unemployed, in education, retired, } \\
\text { looking after home) }\end{array}$ & $5(33 \%)$ & $6(40 \%)$ & $11(37 \%)$ \\
\hline
\end{tabular}


Table 2 Continued

$\begin{array}{lll}\begin{array}{l}\text { Immediate } \\ (n=15)\end{array} & \begin{array}{l}\text { Delayed } \\ (n=15)\end{array} & \begin{array}{l}\text { Total } \\ (n=30)\end{array}\end{array}$

\begin{tabular}{|c|c|c|c|}
\hline \multicolumn{4}{|l|}{ Marital status (n (\%)) } \\
\hline Married/cohabiting & $11(73 \%)$ & $10(67 \%)$ & $21(70 \%)$ \\
\hline Single & 1 (7\%) & $3(20 \%)$ & $4(13 \%)$ \\
\hline Widowed & $1(7 \%)$ & $1(7 \%)$ & $2(7 \%)$ \\
\hline \multicolumn{4}{|l|}{ Household composition (n (\%)) } \\
\hline \multicolumn{4}{|l|}{ At screening (n (\%)): } \\
\hline $\begin{array}{l}\text { Does not do } 30 \text { mins or more of activity that increases } \\
\text { breathing rate on any days in a typical week }\end{array}$ & $7(47 \%)$ & $6(40 \%)$ & $13(43 \%)$ \\
\hline
\end{tabular}

Percentages rounded to nearest integer, and thus, might not sum exactly to $100 \%$.

†While an APDDS score of $\geq 6$ was an exclusion criterion this person's APDDS score at screening was $<6$.

Pain in leg when standing on balance board during Segway Circuit (Training Plus), overdoing it on Hula Hoop (Aerobics) and aggravating existing scar tissue, hurt back during Jackknife (Strength), discomfort in hand when doing the Warrior (Yoga), aggravating an existing knee injury while using the Wii, catching toe on balance board, falling when playing Balance Bubble (Balance), losing footing and near fall with Rhythm Parade (Training Plus), two near falls when doing the Basic Step (Aerobics) and one near fall when using the balance board when playing Perfect 10 (Training Plus).

None of the events was deemed of major concern by the Safety Monitoring Committee and as soon as we became aware of such issues they were followed up promptly by the Mii-vitaliSe facilitators and referred on if necessary. If appropriate, the participant was given advice about how best to adjust their Wii programme to minimise the likelihood of a reoccurrence of an $\mathrm{AE}$.

\section{Use and patterns of use}

Around $20 \%$ of days in the $\log$ and calendar were left blank. We have assumed that the Wii was not used on these days, although this is likely to lead to an underestimation of use. The Wii was used on around $30 \%$ of days during the first 6 months of using the Wii (delayed and immediate groups combined) and 19\% of days in the second 6 months (immediate group). Thirty per cent of days equates to about 2 days per week per person. Median percentage of days used over the first 6 months of having the Wii was $28 \%$ (95\% CI $23 \%$ to $37 \%$ ). In the immediate group at 12 months follow-up, five participants' calendars indicated they were using the Wii at least 20\% of days in the second 6 months. Participants tended to exercise alone, in the morning or afternoon, with light-tomoderate intensity. Balance exercises, yoga and aerobics were the most common activities used. The most frequent reasons for not exercising were no time, too tired and feeling unwell. Descriptive data are presented in Tables S7a \& S7b (see online web supplement 2).

\section{Health economics component}

Healthcare resource use questionnaire

Although this questionnaire states that all boxes should be completed even if the response is zero, participants sometimes left some boxes blank when asked about the number of contacts with various clinicians/health

Table 3 Previous experience/use of technology reported at baseline $(n=30)$

\begin{tabular}{|c|c|c|c|c|c|}
\hline & $\begin{array}{l}\text { Do not } \\
\text { use }\end{array}$ & $\begin{array}{l}\text { Has used but } \\
\text { no longer uses }\end{array}$ & $\begin{array}{l}\text { Uses } \\
\text { occasionally }\end{array}$ & $\begin{array}{l}\text { Uses } \\
\text { regularly }\end{array}$ & $\begin{array}{l}\text { Unsure } \\
\text { what this is }\end{array}$ \\
\hline TV remote control & - & - & $1(3 \%)$ & $29(97 \%)$ & - \\
\hline SMART phone & $14(47 \%)$ & - & - & $16(53 \%)$ & - \\
\hline Sat Nav & $20(67 \%)$ & $1(3 \%)$ & $5(17 \%)$ & $4(13 \%)$ & - \\
\hline Touch screen tablet (eg, iPad) & $14(47 \%)$ & $1(3 \%)$ & $6(20 \%)$ & $9(30 \%)$ & - \\
\hline Handheld gaming system (eg, Nintendo DS) & $22(73 \%)$ & $7(23 \%)$ & $1(3 \%)$ & - & - \\
\hline Home gaming system (eg, Nintendo Wii, Xbox, etc) & $18(60 \%)$ & $8(27 \%)$ & $4(13 \%)$ & - & - \\
\hline
\end{tabular}


Table 4 Descriptives and preliminary estimates of effect size (Cohen's $d$ ) for candidate outcomes for the future trial

\begin{tabular}{|c|c|c|}
\hline $\begin{array}{l}\text { Means (SD) } \mathbf{n} \\
\text { Numbers in bold indicat } \\
{ }^{*} \text { Numbers presented if } 0\end{array}$ & $\begin{array}{l}\text { te measurements taken } \\
\text { different from those in }\end{array}$ & $\begin{array}{l}\text { using Wii } \\
\text { SORT diagram }\end{array}$ \\
\hline Baseline $(T=0)$ & 6 months $(T=1)$ & 12 months ( $T=2)$ \\
\hline $\begin{array}{l}\text { Neither group using Wii } \\
n=30\end{array}$ & $\begin{array}{l}\text { Only immediate group } \\
\text { using Wii } n=29\end{array}$ & $\begin{array}{l}\text { Both groups using } \\
\text { Wii } n=28\end{array}$ \\
\hline
\end{tabular}

Godin Leisure-Time Exercise Questionnaire (higher scores indicate more activity)

Imm (Wii after $\mathrm{T}=0$ )

Delayed (Wii after T=1)

Mean difference adjusted for baseline $(95 \% \mathrm{Cl})$

Standardised effect size

Overall baseline SD $(95 \% \mathrm{Cl})$
$11.07(12.39) 14$
$7.93(11.43) 14$

22.46 (16.39) 13

$11.20(9.77) 15$

$8.32(-2.01$ to 18.65$)$

0.70

11.81 (9.33 to 16.07 ) -

Multiple Sclerosis Self-Efficacy scale (higher scores more self-efficacy, potential range 0-12)
Imm (Wii after $\mathrm{T}=0$ )
57 (16)
59 (20)
59 (19)
Delayed (Wii after $\mathrm{T}=1$ )
$62(18)$
58 (16)
60 (16)
Mean difference adjusted for baseline $(95 \% \mathrm{Cl})$
Standardised effect size
4 (-8 to 15$)$
Overall baseline SD $(95 \% \mathrm{Cl})$
0.23
16.83 (13.40 to 22.62 ) -
$-$
$-$

28.00 (24.61)

18.17 (17.57)

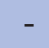

$-$

Hospital Anxiety and Depression Scale-depression subscale (sum score) (higher scores more depression, potential range 0-21)
Imm (Wii after $\mathrm{T}=0)$
$5.70(2.88)$
$5.14(2.96)$
$4.96(2.55)$
Delayed (Wii after $\mathrm{T}=1)$
$6.27(2.84)$
$6.53(2.75)$
$5.93(3.84)$
Mean difference adjusted for baseline $(95 \% \mathrm{Cl})$
Standardised effect size
$-0.67(-2.23$ to 0.88$)$
$-0.23$
Overall baseline SD $(95 \% \mathrm{Cl})$
2.82 (2.25 to 3.80$)$

Hospital Anxiety and Depression Scale-anxiety subscale (sum score) (higher scores more anxiety, potential range 0-21)

Imm (Wii after $\mathrm{T}=0$ )

$8.53(3.62)$

8.07 (3.83)

$7.31(2.93)$

Delayed (Wii after T=1)

$6.27(3.28)$

$6.60(3.81)$

$6.20(4.93)$

Mean difference adjusted for baseline $(95 \% \mathrm{Cl})$

Standardised effect size

Overall baseline SD $(95 \% \mathrm{Cl})$

$-0.41(-2.39$ to 1.58$)$

$-0.12$

3.59 (2.86 to 4.82 )

Spinal Cord Injury Exercise Self-Efficacy Scale (sum score) (higher scores more self-efficacy, potential range 10-40)

Imm (Wii after $\mathrm{T}=0$ )

Delayed (Wii after $T=1$ )

Mean difference adjusted for baseline $(95 \% \mathrm{Cl})$

Standardised effect size

Overall baseline SD $(95 \% \mathrm{Cl})$
$28.47(6.63)$

$28.33(5.37)$

$-$

5.93 (4.72 to 7.97$)$
28.89 (4.96)

$26.27(5.08)$

$2.47(-0.59$ to 5.52$)$

0.41

$28.04(6.62)$

$29.93(5.42)$

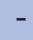

Multiple Sclerosis Impact Scale-29 physical subscale (higher scores more impact (worse outcomes), potential range 0-100)

Imm (Wii after $\mathrm{T}=0$ )

Delayed (Wii after T=1)

Mean difference adjusted for baseline $(95 \% \mathrm{Cl})$

Standardised effect size

Overall baseline SD $(95 \% \mathrm{Cl})$
$30.92(9.37)$

$28.91(11.61)$

$-$

$10.42(8.30$ to 14.00$)$
30.40 (11.09)

30.38 (13.75)

$30.58(14.86)$

31.88 (19.36)

$-1.89(-7.89$ to 4.11$)$

$-0.18$

Multiple Sclerosis Impact Scale-29 psychological subscale (higher scores more impact (worse outcomes), potential range 0-100)

Imm (Wii after $\mathrm{T}=0$ )

Delayed (Wii after T=1)

$30.65(17.45)$

26.29 (18.27)

30.37 (14.38)

25.59 (16.06)

28.17 (18.45) 


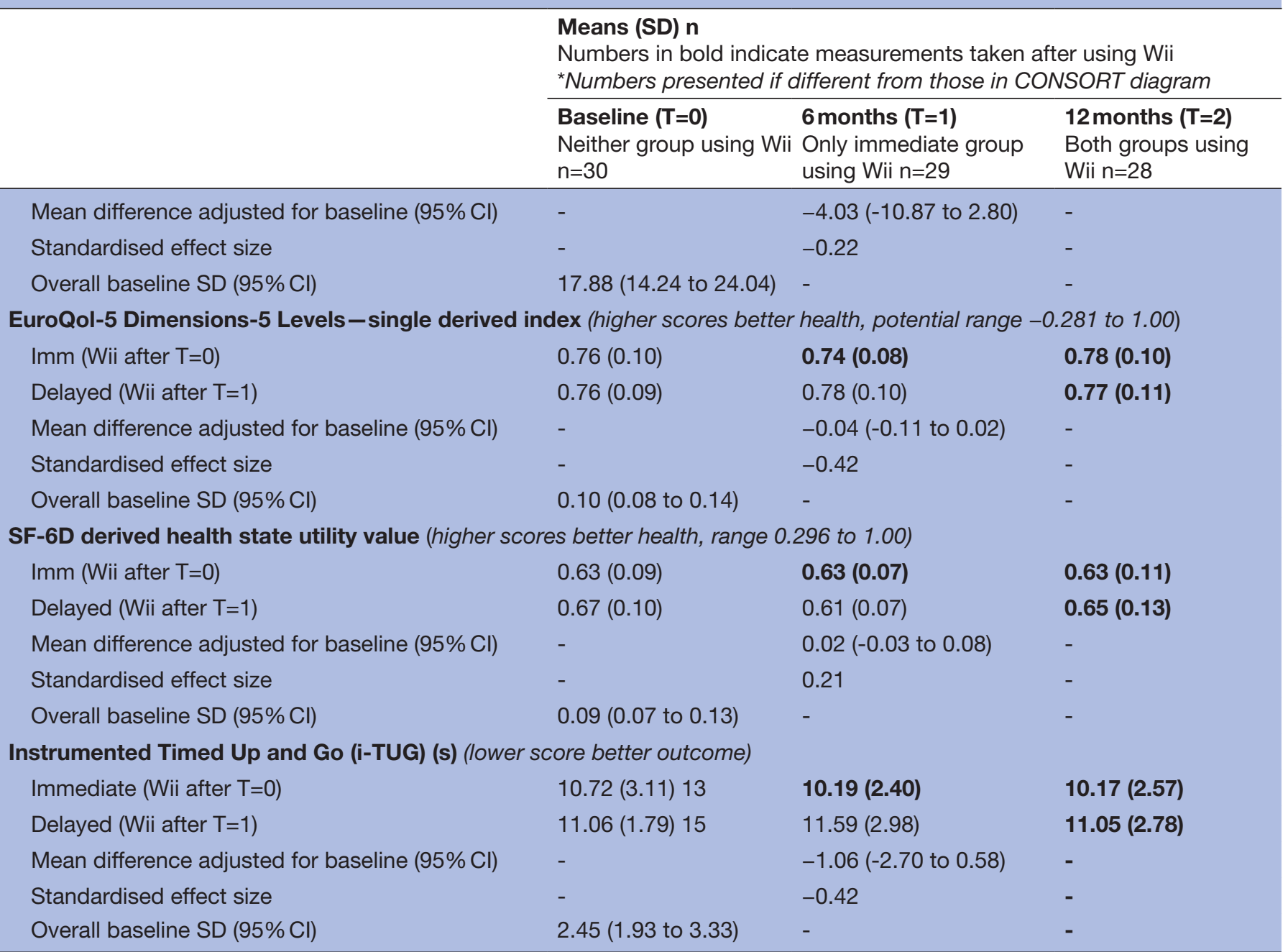

Where high scores indicate better outcomes, positive effect sizes suggest benefit from the Wii. Where low scores indicate better outcomes, negative effect sizes suggest benefit from the Wii. Ns are presented if they differ from ns described.

professionals (10/57 (18\%) of follow-up questionnaires). In these instances when participants had completed some categories but left some blank, we assumed they meant zero. In a future trial as we now have a better idea of the main resources used (and can draw on data from another full-scale trial we have conducted that used the same resource use questionnaire ${ }^{71}$ ), we will be able to reduce the number of categories that we include and hopefully reduce the number of blank fields. Descriptive data are presented in Tables S8a \& S8b (see online web supplement 2).

\section{Intervention costs}

The work sampling forms suggested that the mean time per participant spent by physiotherapists delivering Mii-vitaliSe was 12 hours with approximately half this time involving face-to-face contact (see Table S9, online web supplement 2). Using an estimated cost of $£ 32$ per hour ${ }^{72}$ for a hospital physiotherapist equates to a per participant cost of the intervention of $£ 384$ for physiotherapy time. The equipment cost (Nintendo Wii console plus peripherals and software) was approximately $£ 300$ per unit.
Thus, the estimated total cost of providing Mii-vitaliSe is $£ 684$ per person.

\section{Acceptability of study design}

While some participants felt disappointed on learning that they had been randomised to the delayed (6-month wait) group, this design was generally deemed acceptable. In a definitive trial, this timeframe would limit scope for long-term follow-up. However, a 12-month delay was considered a long time to wait and it was felt this could deter people from wanting to take part. When we explored a design that involved a usual care comparator most said they would still be willing to participate in such a study as they were keen to help others and saw randomisation as 'part and parcel' of clinical trials.

\section{Acceptability of the Mii-vitaliSe intervention-participants}

Most study participants were positive about Mii-vitaliSe, which was seen to be an acceptable, fun and convenient way to increase physical activity levels. A couple of people found the Wii was not for them 
despite initial enthusiasm about joining the study. The materials were seen to be comprehensive and helpful with people tending to use them most when they were first familiarising themselves with the Wii. Physiotherapist contact time was considered to be about right by the majority of participants, was highly valued and considered important to ensure people were safe and adequately supported.

Participants reported a wide range of benefits related to both physical and mental health (eg, increased physical activity, improved mood, reduced stress, increased confidence, better sleep, improved balance, strength and posture, better coordination, improvements in walking and relief in symptoms such as pain and fatigue). Examples of improvements noticed in everyday life included: fewer pegs dropped when hanging out the washing, finding it easier to climb out of a shower, being able to dance more in time to music at a social event, greater accuracy and dexterity when reaching for door handles, being able to walk further. Some people reported that relatives/ friends had noticed improvements. For some the Wii appeared to act as a 'gateway' to other physical activities and to greater self-confidence.

\section{Acceptability of Mii-vitaliSe-physiotherapist facilitators}

The two physiotherapist facilitators enjoyed delivering Mii-vitaliSe, liked the collaborative delivery model and found it helpful working with people in their own homes. They were sometimes struck by how much more people were able to do in the familiar surroundings of their own home compared with the hospital setting. While they found the Mii-vitaliSe materials well-written and presented, they felt there was scope for a simplified version combining the guidance book and workbook into one resource. They suggested the following modifications to the intervention: (i) have the facilitators assess people in their own homes prior to the initial orientation session; (ii) deliver the first orientation session in a structured group format; (iii) provide training for facilitators in setting up the Wii equipment with telephone technical support available; (iv) provide fixed time slots for the monthly follow-up calls with participants; (v) provide formal training in motivational interviewing to all facilitators if they do not already have these skills.

\section{DISCUSSION}

The findings suggest that Mii-vitaliSe was well received by the majority of participants and acceptable to the physiotherapists delivering it. Study recruitment was successful, retention was good and attrition low. Participants found the questionnaires relevant and easy to complete (with the exception of the APDDS) and questionnaire completion rates were high. Use of the Wii was good (average of around 2 days per week) and approached aerobic activity guideline levels developed for MS. ${ }^{16}$ This fits in with public health recommendations to encourage modest increases in physical activity in those who are sedentary. ${ }^{17}$

Limitations of this study include that it took place in a single centre linked with a multidisciplinary MS team, which is not typical of services available to patients across the country. Second, administration of the physical outcome assessments was not undertaken by a blinded assessor. Third, males were under-represented. Finally, it is likely that there was a selection bias with those who enrolled in the study more likely to find active gaming appealing.

In terms of strengths, we have developed an intervention in line with the Medical Research Council guidance for the development of complex interventions ${ }^{74}$ that blends physiotherapy and psychological approaches and with substantial service user input. The intervention incorporates behaviour change techniques and the study included mixed methods and a consideration of long-term follow-up.

While the current study involved use of the Nintendo Wii, technology moves at a swift pace and the Wii has since been superseded by the Wii U (which itself recently ceased production). The Wii U offers certain benefits in terms of its capability to support remote communication (via 'Miiverse') interaction, and competition. It enables the user to set up individualised routines and has a personal trainer feature. Several participants in the current study said in the interviews that they would have liked to have been able to contact others in order to provide mutual encouragement and share tips. Having peer support may help to enhance people's motivation and ongoing use. We conducted usability testing of the Wii $\mathrm{U}$ with four participants from the current study. Overall, they liked the Wii U and felt it had certain advantages including a wider range of games and possibilities for remote communication.

Looking ahead to a future definitive trial, the main contender for a primary outcome is the Godin LeisureTime Exercise Questionnaire. This had the largest standardised effect size and increased physical activity was a key benefit described by participants in the interviews.

Since we designed the protocol for the feasibility study, guidelines for core outcomes to include in exercise trials in people with MS have been published. ${ }^{75}$ Ideally, we would like to avoid or reduce the requirements for people to attend the hospital for the administration of outcome measures as feedback from this feasibility study suggested such visits can be tiring and inconvenient for people who are already experiencing fatigue. While patient-reported questionnaires and accelerometers can be administered postally without requiring trips to the hospital, assessments such as the Timed Up and Go and the Two Minute or Six Minute Walk Test require assessor administration. It would be challenging to undertake these blinded in the home in the context of a randomised trial with a usual care comparator as the Wii equipment might be visible in the homes of those allocated to the Mii-vitaliSe arm. To finalise the outcome set for the future trial, we will consider the most recent literature and consult with service users. 
Our study is the first to report on home-based use of the Wii for people with MS in the UK. Overall, findings from this study are promising and support proceeding to a full-scale trial of effectiveness and cost-effectiveness. We will refine the trial design, aspects of the intervention and finalise outcome measures in the light of our experiences from this pilot study. This work has relevance for the development and evaluation of other interventions that incorporate technology and personalised support to enhance physical activity levels in long-term conditions.

Acknowledgements The authors would like to thank the individuals with MS who attended the consultation workshop to inform the development of the Mii-vitaliSe intervention and all the participants who took part in the study. The authors also thank Dr Kate Jupp and Dr Mark Cossburn for their contributions as members of the study Safety Monitoring Committee.

Contributors ST: Chief Investigator, conception, design of study and intervention materials, analysis, interpretation, produced initial draft of manuscript. LF: design of intervention materials, acquisition of data, undertook interviews, analysis of assessment data, interpretation; usability testing for Wii U. PT: conception, design of study, advised on analysis, interpretation. SC, SB: clinical aspects of design, provided feedback on intervention materials, delivered intervention. $\mathrm{CH}$ : clinical aspects of study design, oversaw clinical aspects. SP: design of gait and stride physical assessments, acquisition of gait/stride data, advised on analysis, interpretation. RS: contributed to technology-related aspects of study design and provided advice during study. FT: analysis of physical activity and adherence data and interpretation. Undertook usability testing for Wii U. CT: undertook usability testing for Wii U. KJ, JH provided feedback about the draft Mii-vitaliSe materials and the physical assessment protocol and provided advice throughout the study from a service user perspective. LF, PT and FT contributed to draft manuscript and all other authors critically reviewed manuscript and approved the final version.

Funding This study was funded by a project grant awarded by the MS Society in the UK (ref no. 933/10). It is included in the National Institute of Health Research Clinical Research Network (NIHR CRN) portfolio (ID 13130).

Competing interests None declared.

Ethics approval National Health Service (NHS) South Central Hampshire B Research Ethics Committee (ref: 12/SC/0420)

Provenance and peer review Not commissioned; externally peer reviewed.

Data sharing statement All available data are reported.

Open Access This is an Open Access article distributed in accordance with the Creative Commons Attribution Non Commercial (CC BY-NC 4.0) license, which permits others to distribute, remix, adapt, build upon this work non-commercially, and license their derivative works on different terms, provided the original work is properly cited and the use is non-commercial. See: http://creativecommons.org/ licenses/by-nc/4.0/

(c) Article author(s) (or their employer(s) unless otherwise stated in the text of the article) 2017. All rights reserved. No commercial use is permitted unless otherwise expressly granted.

\section{REFERENCES}

1. Rietberg MB, Brooks D, Uitdehaag BMJ, et al. Exercise therapy for multiple sclerosis. Cochrane Database Syst Rev 2004;3:CD003980.

2. Learmonth YC, Motl RW. Physical activity and exercise training in multiple sclerosis: a review and content analysis of qualitative research identifying perceived determinants and consequences. Disabil Rehabil 2016;38:1227-42.

3. Snook EM, Motl RW. Effect of exercise training on walking mobility in multiple sclerosis: a meta-analysis. Neurorehabil Neural Repair 2009;23:108-16.

4. Pilutti LA, Greenlee TA, Motl RW, et al. Effects of exercise training on fatigue in multiple sclerosis: a meta-analysis. Psychosom Med 2013;75:575-80.

5. Latimer-Cheung AE, Pilutti LA, Hicks AL, et al. Effects of exercise training on fitness, mobility, fatigue, and health-related quality of life among adults with multiple sclerosis: a systematic review to inform guideline development. Arch Phys Med Rehabil 2013;94:1800-28.

6. Motl RW, Gosney JL. Effect of exercise training on quality of life in multiple sclerosis: a meta-analysis. Mult Scler 2008;14:129-35.

7. Ensari I, Motl RW, Pilutti LA. Exercise training improves depressive symptoms in people with multiple sclerosis: results of a metaanalysis. J Psychosom Res 2014;76:465-71.

8. Pilutti LA, Platta ME, Motl RW, et al. The safety of exercise training in multiple sclerosis: a systematic review. J Neurol Sci 2014;343:3-7.

9. Beckerman H, de Groot V, Scholten MA, et al. Physical activity behavior of people with multiple sclerosis: understanding how they can become more physically active. Phys Ther 2010;90:1001-13.

10. Asano M, Duquette P, Andersen R, et al. Exercise barriers and preferences among women and men with multiple sclerosis. Disabil Rehabil 2013;35:353-61.

11. Stroud N, Minahan C, Sabapathy S. The perceived benefits and barriers to exercise participation in persons with multiple sclerosis. Disabil Rehabil 2009;31:2216-22.

12. Borkoles E, Nicholls AR, Bell K, et al. The lived experiences of people diagnosed with multiple sclerosis in relation to exercise. Psychol Health 2008;23:427-41.

13. Kayes NM, McPherson KM, Taylor D, et al. Facilitators and barriers to engagement in physical activity for people with multiple sclerosis: a qualitative investigation. Disabil Rehabil 2011;33:625-42.

14. Goble DJ, Cone BL, Fling BW. Using the Wii Fit as a tool for balance assessment and neurorehabilitation: the first half decade of "Wiisearch". J Neuroeng Rehabil. In Press. 2014;11:12.

15. Warburton DER. The health benefits of active gaming: separating the myths from the virtual reality. Curr Cardiovasc Risk Rep 2013;7:251-5.

16. Latimer-Cheung AE, Martin Ginis KA, Hicks AL, et al. Development of evidence-informed physical activity guidelines for adults with multiple sclerosis. Arch Phys Med Rehabil 2013;94:1829-36.

17. de Souto Barreto P. Global health agenda on non-communicable diseases: has WHO set a smart goal for physical activity? BMJ 2015;350:h23.

18. Ravenek KE, Wolfe DL, Hitzig SL. A scoping review of video gaming in rehabilitation. Disabil Rehabil Assist Technol 2016;11:445-53.

19. Donzé C. Update on rehabilitation in multiple sclerosis. Presse Med 2015;44:e169-e176.

20. Taylor MJ, Griffin M. The use of gaming technology for rehabilitation in people with multiple sclerosis. Mult Scler 2015;21:355-71.

21. Plow M, Finlayson M. Potential benefits of Nintendo Wii Fit among people with multiple sclerosis. Int J MS Care 2011;13:21-30.

22. Brichetto $G$, Spallarossa $P$, de Carvalho ML, et al. The effect of Nintendo $\AA$ Wii $\AA$ on balance in people with multiple sclerosis: a pilot randomized control study. Mult Scler 2013;19:1219-21.

23. Nilsagård YE, Forsberg AS, von Koch L. Balance exercise for persons with multiple sclerosis using Wii games: a randomised, controlled multi-centre study. Mult Scler 2013;19:209-16.

24. Guidi I, Giovannelli T, Paci M. Effects of Wii exercises on balance in people with multiple sclerosis. Mult Scler 2013;19:965.

25. Robinson J, Dixon J, Macsween A, et al. The effects of exergaming on balance, gait, technology acceptance and flow experience in people with multiple sclerosis: a randomized controlled trial. BMC Sports Sci Med Rehabil 2015;7:8.

26. Prosperini L, Fortuna D, Giannì C, et al. Home-based balance training using the Wii balance board: a randomized, crossover pilot study in multiple sclerosis. Neurorehabil Neural Repair 2013;27:516-25.

27. Forsberg A, Nilsagård $Y$, Boström K. Perceptions of using videogames in rehabilitation: a dual perspective of people with multiple sclerosis and physiotherapists. Disabil Rehabil 2015;37:338-44.

28. Kramer A, Dettmers C, Gruber M. Exergaming with additional postural demands improves balance and gait in patients with multiple sclerosis as much as conventional balance training and leads to high adherence to home-based balance training. Arch Phys Med Rehabil 2014;95:1803-9.

29. Bonnechère $B$, Jansen $B$, Omelina $L$, et al. The use of commercial video games in rehabilitation: a systematic review. Int $J$ Rehabil Res 2016;39:277-90.

30. Lieberman DA, Chamberlin B, Medina E, et al. The power of play: innovations in getting Active Summit 2011: a science panel proceedings report from the American Heart Association. Circulation 2011;123:2507-16.

31. Plow M, Finlayson M. A qualitative study exploring the usability of Nintendo Wii Fit among persons with multiple sclerosis. Occup Ther Int 2014;21:21-32. 
32. Plow MA, McDaniel C, Linder S, et al. Scoping review of exergaming for adults with systemic disabling conditions. J Bioeng Biomed Sci 2011;30:559-76.

33. Sangelaji B, Smith CM, Paul L, et al. The effectiveness of behaviour change interventions to increase physical activity participation in people with multiple sclerosis: a systematic review and metaanalysis. Clin Rehabil 2016;30:559-76.

34. Learmonth YC, Adamson BC, Balto JM, et al. Multiple sclerosis patients need and want information on exercise promotion from healthcare providers: a qualitative study. Health Expect 2016:1-10.

35. Thomas S, Fazakarley L, Thomas PW, et al. Testing the feasibility and acceptability of using the Nintendo Wii in the home to increase activity levels, vitality and well-being in people with multiple sclerosis (Mii-vitaliSe): protocol for a pilot randomised controlled study. BMJ Open 2014;4:e005172.

36. Eldridge SM, Chan CL, Campbell MJ, et al. CONSORT 2010 statement: extension to randomised pilot and feasibility trials. BMJ 2016;355:i5239.

37. Orme M, Kerrigan J, Tyas D, et al. The effect of disease, functional status, and relapses on the utility of people with multiple sclerosis in the UK. Value Health 2007;10:54-60.

38. Lancaster GA, Dodd S, Williamson PR. Design and analysis of pilot studies: recommendations for good practice. J Eval Clin Pract 2004; 10:307-12.

39. Thomas S, Fazakarley L, Thomas PW, et al. Mii-vitaliSe Development of a physiotherapist-supported Nintendo Wii (TM) intervention to encourage people with multiple sclerosis to become more active in the home. MSJ 2015;21:775.

40. Miller WR, Rollnick S. Motivational Interviewing: Preparing People to Change. 2nd ed. NY: Guilford Press, 2002. ISBN 9781572305632.

41. Bandura A. Social learning theory. New York: General Learning Press, 1971.

42. Bandura A, A social-cognitive theory of personality. Pervin $L$ John $O$, ed. Handbook ofpersonality: theory and research. 2nd ed. New York: Guilford Press, 1999: 154.

43. Bandura A. Self-efficacy: the exercise of self-control. NY: Freeman 1997.

44. Beck A. Cognitive therapy: a thirty years retrospective. Am Psychol 1991;466:368-75.

45. Ryan RM, Deci EL. Self-determination theory and the facilitation of intrinsic motivation, social development, and well-being. Am Psychol 2000;55:68-78.

46. Godin G, Shephard RJ. A simple method to assess exercise behavior in the community. Can J App/ Sport Sci 1985;10:141-6.

47. Gosney JL, Scott JA, Snook EM, et al. Physical activity and multiple sclerosis: validity of self-report and objective measures. Fam Community Health 2007;30:144-50.

48. Ryan CG, Grant PM, Tigbe WW, et al. The validity and reliability of a novel activity monitor as a measure of walking. $\mathrm{Br} J$ Sports Med 2006;40:779-84.

49. Edwardson $\mathrm{CL}$, Winkler EAH, Bodicoat $\mathrm{DH}$, et al. Considerations when using the activPAL considerations when using the activPAL monitor in field based research with adult populations. J Sport Health Sci 2016.

50. Zigmond AS, Snaith RP. The hospital anxiety and depression scale. Acta Psychiatr Scand 1983;67:361-70.

51. EuroQol Group. EuroQol--a new facility for the measurement of health-related quality of life. Health Policy 1990;16:199-208.

52. Devlin N, Shah N, Feng N, Mulhern N, van Hout N. Valuing healthrelated quality of life: an EQ-5D-5L value set for England. Office of Health Economics: London, UK, 2016.

53. Hobart J, Lamping D, Fitzpatrick R, et al. The multiple sclerosis impact Scale (MSIS-29): a new patient-based outcome measure. Brain 2001;124:962-73.
54. Hann DM, Jacobsen PB, Azzarello LM, et al. Measurement of fatigue in Cancer patients: development and validation of the Fatigue Symptom Inventory. Qual Life Res 1998;7:301-10.

55. Ware JE, Sherbourne CD. The MOS 36 -item short-form health survey (SF-36). I. Conceptual framework and item selection. Med Care 1992;30:473-83.

56. Brazier JE, Roberts J. The estimation of a preference-based measure of health from the SF-12. Med Care 2004;42:851-9.

57. Kroll T, Kehn M, Ho PS, et al. The SCI Exercise Self-Efficacy Scale (ESES): development and psychometric properties. Int J Behav Nutr Phys Act 2007;4:34.

58. Schwartz CE, Coulthard-Morris L, Zeng Q, et al. Measuring selfefficacy in people with multiple sclerosis: a validation study. Arch Phys Med Rehabil 1996;77:394-8.

59. Rossier P, Wade DT. Validity and reliability comparison of 4 mobility measures in patients presenting with neurologic impairment. Arch Phys Med Rehabil 2001;82:9-13.

60. Hill KD, et al. A New Test of Dynamic standing balance for Stroke Patients: reliability, validity and comparison with healthy elderly. Physiotherapy Canada 1996;48:257-62.

61. Frzovic D, Morris ME, Vowels L. Clinical tests of standing balance: performance of persons with multiple sclerosis. Arch Phys Med Rehabil 2000;81:215-21.

62. Cattaneo D, Regola A, Meotti M. Validity of six balance disorders scales in persons with multiple sclerosis. Disabil Rehabil 2006;28:789-95.

63. Perring S, Summers T. Laboratory-free measurement of gait rhythmicity in the assessment of the degree of impairment and the effectiveness of rehabilitation in patients with Vertigo resulting from vestibular hypofunction. Physiol Meas 2007;28:697-705.

64. Perring S, Jepson R. The use of a two-dimensional ultrasound timeof-flight system for location of the centre of gravity in posturography measurements. Physiol Meas 2001;22:447-52.

65. EquiTest Operator's Manual, version 4.02, Appendix F: Technical Information. Clackamas, Oregon: NeuroCom International, 1992.

66. Llorens R, Latorre J, Noé E, et al. Posturography using the Wii Balance Board ${ }^{\mathrm{TM}}$ : a feasibility study with healthy adults and adults post-stroke. Gait Posture 2016;43:228-32.

67. Severini G, Straudi S, Pavarelli C, et al. Use of Nintendo Wii Balance Board for posturographic analysis of multiple sclerosis patients with minimal balance impairment. J Neuroeng Rehabil 2017;14:19.

68. Mathiowetz V, Weber K, Kashman N, et al. Adult norms for the nine Hole Peg Test of Finger Dexterity. OTJR 1985;5:24-38.

69. Norton K, Norton L, Sadgrove D. Position statement on physical activity and exercise intensity terminology. J Sci Med Sport 2010;13:496-502.

70. Borg GA. Psychophysical bases of perceived exertion. Med Sci Sports Exerc 1982;14:377-81.

71. Thomas S, Thomas PW, Kersten P, et al. A pragmatic parallel arm multi-centre randomised controlled trial to assess the effectiveness and cost-effectiveness of a group-based fatigue management programme (FACETS) for people with multiple sclerosis. $J$ Neurol Neurosurg Psychiatry 2013:84:1092-9.

72. Curtis L. The unit costs of health and social care 2013. Kent: Personal Social Services Research Unit, 2013. Available at: http:// www.pssru.ac.uk/project-pages/unit-costs/2013/.

73. Gale NK, Heath G, Cameron E, et al. Using the framework method for the analysis of qualitative data in multi-disciplinary health research. BMC Med Res Methodol 2013;13:117.

74. Craig P, Dieppe P, Macintyre S, et al. Developing and evaluating complex interventions: the New Medical Research Council guidance. BMJ 2008;337:a1655.

75. Paul L, Coote S, Crosbie J, et al. Core outcome measures for exercise studies in people with multiple sclerosis: recommendations from a multidisciplinary consensus meeting. Mult Scler 2014;20:1641-50. 